\title{
Anti-CD20 monoclonal antibody (rituximab) treatment for cryoglobulinemic vasculitis: where do we stand?
}

\author{
P Cacoub, A Delluc, D Saadoun, D A Landau, D Sene
}

Université Pierre et Marie Curie Paris 6, CNRS, UMR 7087, Paris, France; AP-HP, Hôpital Pitié-

Salpêtrière, Service de Médecine Interne, Paris, France

Correspondence to:

Professor P Cacoub, AP-HP, Hôpital Pitié-Salpêtrière, Service de Médecine Interne, Paris, F75013 France; patrice.cacoub@ psl.aphp.fr

Accepted 28 June 2007 Published Online First 20 July 2007

\section{ABSTRACT}

Mixed cryoglobulinemia (MC) vasculitis represents a complication of the B cell response to a variety of chronic inflammatory diseases. Recent reports describe the use of monoclonal antibodies directed to CD20 antigen (rituximab), a transmembrane protein expressed on pre-B lymphocytes and mature lymphocytes. The goal of this article is therefore to review published data in order to better analyse the efficacy and tolerance of rituximab treatment in patients with MC vasculitis. After systematic review of the literature and exclusion of review papers, 13 manuscripts were identified that reported on a total number of 57 cases of MC secondary to hepatitis $C$ virus (HCV) infection (75.4\%) or essential mixed cryoglobulinemia (24.6\%). Previous treatments failed to control the main signs of vasculitis; these were either HCV $(n=37)$ or immunomodulating treatments. Most patients (48 out of 57) received four weekly consecutive intravenous infusions of $375 \mathrm{mg} / \mathrm{m}^{2}$ of rituximab. The duration of follow-up after rituximab therapy was 9.7 months. Rituximab infusions had great efficacy on the main vasculitis signs, with a clinical response in $80-93 \%$ patients. A relapse of $\mathrm{MC}$ was noted in 14 out of 36 (39\%) patients. A relatively small number of side effects were reported. We conclude that rituximab therapy for patients with mixed cryoglobulinemia vasculitis, HCVinduced or essential, shows great efficacy on the main vasculitis signs in the majority of reported patients. A relapse of cryoglobulinemia vasculitis was frequently noted. Randomised controlled trials with long-term study are needed to form definitive conclusions on the benefit/ risk ratio of rituximab therapy in such patients.

Mixed cryoglobulinemia (MC) is a systemic vasculitis characterised by the proliferation of B-cell clones producing pathogenic IgM with rheumatoid factor activity. MC leads to clinical manifestations ranging from MC syndrome (purpura, arthralgia, asthenia) to more serious lesions with neurological and renal involvement. ${ }^{12}$ Since 1990, it has been established that hepatitis C virus (HCV) infection is associated with most cases of MC. On the one hand, 60-90\% of patients with $\mathrm{MC}$ are $\mathrm{HCV}$-infected. On the other, $36-55 \%$ of HCV-infected patients have a positive mixed cryoglobulin. Up to $15-20 \%$ of HCV-MC patients will present a MC-associated systemic vasculitis that may be severe enough to cause death. ${ }^{34}$

Limited data are available regarding the treatment of patients with MC systemic vasculitis. In patients with HCV infection, interferon (IFN)- $\alpha$ monotherapy is associated with a relatively poor response and a high relapse rate, especially in severe cases. ${ }^{56}$ The combination of pegylated IFN $\alpha$ (pegIFN $\alpha$ ) with ribavirin, which is the current standard for the treatment of patients with chronic HCV infection, has shown better efficacy, with up to $77 \%$ of patients showing complete remission. The poor tolerability (in many cases) of conventional anti-HCV therapy, including flu-like symptoms, lends further importance to the development of additional therapeutic approaches. The use of corticosteroids, cyclophosphamide, and plasmapheresis, both in HCV-MC patients with no response or intolerance to $\mathrm{HCV}$ treatment and in patients with non-HCV MC, may lead to lifethreatening complications and is difficult to manage in the long term.

After initial single case reports, ${ }^{8-10}$ two Italian groups have reported the efficacy of anti-CD20 monoclonal antibody (rituximab) treatment in patients with HCV-MC vasculitis resistant or intolerant to IFN- $\alpha$ monotherapy. ${ }^{11}{ }^{12}$ Such approach involves the use of monoclonal antibodies directed to CD20 antigen, a transmembrane protein expressed on pre-B lymphocytes and mature B lymphocytes. Rituximab proved effective on skin vasculitis manifestations, subjective symptoms of peripheral neuropathy, arthralgia and low grade B-cell lymphoma. Most clinical responders also saw a decrease in serum cryoglobulin levels and increased in C4 serum levels, though not to undetectable or normal levels. However, one potential concern regarding the use of rituximab is its propensity to worsen $\mathrm{HCV}$ viremia, ${ }^{12}$ which may lead patients to develop more severe HCVinduced liver lesions and/or cryoglobulinemic relapses in subsequent years. ${ }^{13}$ These studies did not allow conclusions to be drawn concerning the efficacy of anti-CD20 monoclonal antibody on peripheral neuropathy and nephropathy.

The goal of this article is to review published data in order to better analyse the efficacy and tolerance of rituximab treatment in patients with cryoglobulinemia vasculitis.

\section{METHODS}

A systematic PubMed search with the key words "cryoglobulinemia", "rituximab" and "anti-CD20" resulted in a list of 54 manuscripts. Review papers were excluded. A total of 15 manuscripts that reported on at least one case of cryoglobulinemia vasculitis treated with rituximab were included in the present analysis. ${ }^{8-22}$ Two manuscripts were excluded because they included initial reports of patients that had already been reported on in other publications. $^{89}$
This paper is freely available
online under the BMJ Journals unlocked scheme, see http:// ard.bmj.com/info/unlocked.dtl 
The response to treatment was analysed by comparing clinical and immunological parameters before rituximab infusion and at the end of follow-up. Clinical response was defined by analysing the evolution of the following main clinical signs: skin involvement (absence of purpura), peripheral neuropathy (clinical and/or electrophysiological improvement), renal involvement (normalisation of serum creatinine level and disappearance of proteinuria), and the absence of arthralgia. Response to rituximab treatment was defined as complete (disappearance of baseline manifestations) or partial (improvement of baseline clinical manifestations); all other patients were classified as nonresponders. Relapse was defined as the reappearance of at least one clinical sign of vasculitis. A complete immunological response was defined by the absence of serum cryoglobulin, and a partial immunological response was defined by a decrease of more than $50 \%$ from the baseline cryoglobulin level.

\section{RESULTS}

Overall, 13 manuscripts reported on a total number of 57 cases that were analysed in detail for the present study. ${ }^{10-23}$ There were two large uncontrolled series of $20^{12}$ and $15^{11}$ patients, and two smaller series of $6^{13}$ and $5^{21}$ patients. All other publications reported either single or two case reports.

The baseline characteristics of patients with cryoglobulinemia vasculitis who received anti-CD20 antibody (rituximab) treatment are summarised in table 1 . A total of 57 patients, mainly of female gender (79\%) with a mean age of 59 years, had cryoglobulinemia vasculitis secondary to chronic active HCV infection $(75.4 \%)$ or essential mixed cryoglobulinemia (24.6\%). Previous treatments failed to control the main signs of vasculitis; these were either HCV treatment $(n=37)$ or immunomodulating treatments (corticosteroids, immunosuppressive drugs, plasma exchanges). The main clinical manifestations of cryoglobulinemia vasculitis were skin involvement (84.2\%), arthralgia (61.4\%), peripheral neuropathy $(54.4 \%)$ and glomerulonephritis (31.6\%).

The main indication for rituximab therapy was non-responsiveness to other previous treatments $(n=50)$, intolerance to previous treatments $(n=3)$, associated lymphoma $(n=2)$, or first-line therapy for cryoglobulinemia vasculitis $(n=2)$.

Most patients (48 out of 57) received four weekly consecutive intravenous infusions of $375 \mathrm{mg} / \mathrm{m}^{2}$ of rituximab. In other cases ( 9 out of 57 ), however, the rituximab treatment protocol was different. In the Rocatello et al series, ${ }^{13}$ five out of six patients received four weekly consecutive intravenous infusions of $375 \mathrm{mg} / \mathrm{m}^{2}$ of rituximab followed by two more infusions at months 1 and 2 . In the patient reported by Ghobrial et al, ${ }^{15}$ who also had Waldenström disease, eight weekly consecutive intravenous infusions of $375 \mathrm{mg} / \mathrm{m}^{2}$ of rituximab were given. In the case reported by Koukoulaki et al, ${ }^{19}$ the patient received four weekly consecutive intravenous infusions of $375 \mathrm{mg} / \mathrm{m}^{2}$ of rituximab, followed by two $1 \mathrm{~g}$ infusions at months 4 and 4.5 . In the case reported by Lamprecht et al, ${ }^{14} 500 \mathrm{mg}$ of rituximab was given every 3 weeks for a total of six infusions. The patient of Ghijsels et $a 1^{16}$ received four weekly consecutive intravenous infusions of $375 \mathrm{mg} / \mathrm{m}^{2}$ of rituximab, followed by a second series of four courses of rituximab at the same dosage 2 months later.

The mean duration of follow-up after rituximab therapy was 9.7 months ( 0.3 to 24$)$. The evolution of the cryoglobulinemia vasculitis features after rituximab infusions are given in table 2. Rituximab infusions had great efficacy on the main vasculitis signs, with a clinical response (partial + complete/total) in 32 out of $40(80 \%)$ patients for skin involvement, 27 out of 34
(79.4\%) for arthralgia, 27 out of 29 (93.1\%) for neuropathy, and 15 out of 18 (83.3\%) for glomerulonephritis. However, a relapse of cryoglobulinemia vasculitis was noted in 14 out of 36 (39\%) patients (13 HCV-infected, $1 \mathrm{HCV}$ negative) within a few days to 19 months (mean 6.7 months) after the last rituximab infusion. Eight of the 14 relapse patients had complete remission after a second course of rituximab. Baseline plus follow up HCV viral load was available in only five reported patients, whereas in the large study of Sansonno the mean HCV viral load increased at month 6 and month $12 .{ }^{16}$ There was no significant difference in the efficacy of rituximab therapy when patients presented with HCV-induced or essential cryoglobulinemia vasculitis (table 3 ).

A relatively small number of side effects were reported. During the short term follow-up they included: bradycardia (3), hypotension (2), infection (in three renal transplant patients), mild alanine aminotransferase (ALT) elevation (3), retinal arterial thrombosis (1), panniculitis of elbows and knees (1), and serum sickness (1). Two deaths were reported; one occurred 12 months after rituximab infusion in an HCV-infected patient with renal insufficiency, and the second occurred 2 months after rituximab infusion in an HCV-negative renal transplant patient due to Cryptococcus neoformans meningoencephalitis. ${ }^{18}$ During the long-term follow-up ( $>12$ months), there were two cases of lymphoma and one of breast cancer. ${ }^{11}$

\section{DISCUSSION}

Although this literature review does not allow a definite conclusion to be drawn regarding the role of rituximab in the therapeutic options of patients presenting with cryoglobulinemia vasculitis, it gives important information concerning its efficacy and short-term tolerance. When we analysed the efficacy, a complete response was reported in one-third to two-thirds of patients, depending on the main targeted organ (skin, nerve, kidney, joint). Complete plus partial responses were noted in $80-93 \%$ of reported patients. Such results are encouraging considering that most patients presented with a very severe form of systemic cryoglobulinemia vasculitis that was resistant or intolerant to other previous treatments. Such a disease remains life threatening, with a mortality rate ranging from $15-20 \%$ in HCV-MC patients and up to $50 \%$ in non-HCVMC patients. ${ }^{12}$ The main predictors of poor outcome are older age and renal insufficiency (present in one-third of the cases treated with rituximab).

Most reported cases (75\%) were secondary to HCV infection. There has been some debate about the best therapeutic management of HCV MC patients (ie, anti-HCV, immunomodulating drugs or both). ${ }^{6}$ It is noteworthy that in one of the two large open studies using rituximab, ${ }^{12} \mathrm{HCV}$ MC patients were described as "non-responders or intolerant to interferon $\alpha$ monotherapy". Such treatment, however, is known to have a poor response on the main symptoms of cryoglobulinemia vasculitis (ie, less than 10\% except for purpuric skin lesions). ${ }^{5}{ }^{6}$ This poor efficacy is related to the low percentage (less than $15 \%$ ) of long term sustained viral clearance. More recent data with the best available HCV treatment (peg-IFN $\alpha$ plus ribavirin), which results in a sustained viral clearance in up to $55 \%$ of patients, showed much better responses, with a complete clinical response of cryoglobulinemia vasculitis in up to $77 \%$ of patients. ${ }^{7}$ However, the poor tolerability of HCV therapy often leads to decrease the doses or duration of treatment with lower clinical and virological responses. Using rituximab, a clinical response was noted in two-thirds of patients, but $39 \%$ of them developed a relapse after a mean 
Table 1 Main baseline characteristics of patients with cryoglobulinemia vasculitis who received anti-CD20 antibody (rituximab) treatment

\begin{tabular}{|c|c|c|c|}
\hline & $\begin{array}{l}\text { No. of patients with } \\
\text { available data }\end{array}$ & $\begin{array}{l}\text { No. of positive } \\
\text { patients }\end{array}$ & Positive patients (\%) \\
\hline Age (years), mean (range), & 57 & - & $59(31-79)$ \\
\hline $\operatorname{Sex}(f) \%$ & 57 & 45 & 79 \\
\hline \multicolumn{4}{|l|}{ Vasculitis : } \\
\hline Duration (months), mean (range) & 57 & - & $60.1(6-240)$ \\
\hline Skin involvement & 57 & 48 & 84.2 \\
\hline Arthralgia & 57 & 35 & 61.4 \\
\hline Neuropathy & 57 & 31 & 54.4 \\
\hline Glomerulonephritis & 57 & 18 & 31.6 \\
\hline \multicolumn{4}{|l|}{ Immunology: } \\
\hline Cryoglobulin positive & 57 & 57 & 100 \\
\hline Type I & & 2 & 3.5 \\
\hline Type II & & 41 & 71.9 \\
\hline Type III & & 10 & 17.6 \\
\hline Type unknown & & 4 & 7.0 \\
\hline Rheumatoid factor positive & 57 & 30 & 52.6 \\
\hline C4 serum level (mg/dl), mean & 57 & - & 7.1 \\
\hline HCV status & 57 & & \\
\hline HCV RNA negative or unknown & & 14 & 24.6 \\
\hline HCV RNA positive & & 43 & 75.4 \\
\hline Genotype 1-4 & & 24 & 55.8 \\
\hline Genotype 2-3 & & 18 & 41.9 \\
\hline Genotype not available & & 1 & 2.3 \\
\hline Viral load $>2$ million IU/mL & 8 & 6 & 75.0 \\
\hline ALT (IU/L), mean & 31 & - & 54.3 \\
\hline \multicolumn{4}{|l|}{ Previous treatment } \\
\hline HCV infection & 37 & & \\
\hline Interferon $\alpha$ & & 27 & 72.8 \\
\hline Pegylated interferon $\alpha$ plus ribavirin & & 4 & 14.8 \\
\hline None & & 12 & 32.4 \\
\hline \multicolumn{4}{|l|}{ Vasculitis treatment: } \\
\hline Corticosteroids & $36^{*}$ & 31 & 86.1 \\
\hline Immunosupressive drug & 56 & 18 & 32.1 \\
\hline Plasma exchange & 56 & 12 & 21.4 \\
\hline
\end{tabular}

*This number is underestimated because patients reported in the series of Sansonno et al ${ }^{12}$ were not included due to the lack of details ("most patients were previously given low to moderate doses of corticosteroids").

$\mathrm{ALT}$, alanine aminotransferase; HCV, hepatitis C virus.

delay of 6.7 months. In this setting, rituximab cannot be seen as a curative treatment as long as the viral antigenic trigger of the vasculitis remains. A sequential treatment strategy may therefore give better results. This would begin with rituximab to block the B-cell activation and the TH1 cytokine-chemokine cascade involved in the inflammatory part of vasculitis, ${ }^{23}$ and be followed by anti-HCV treatment with peg-IFN $\alpha$ plus ribavirin to clear the antigenic trigger and block the causative agent. ${ }^{12}$ Such a strategy may also allow major side effects due to conventional treatments such as corticosteroids, immunosuppressive drugs and plasma exchanges to be obviated.

Due to the very small number of patients with non-HCV systemic cryoglobulinemia vasculitis, it is difficult to come to a clear conclusion in this setting, although rituximab therapy seems to have similar efficacy on the main involved organs (table 3). This form of the disease is close to non-Hodgkin's lymphoma and may also be sensitive to CD20 blockade. Furthermore, limitations due to HCV disease (viremia) will be absent, suggesting that autoimmune-disease-associated $\mathrm{MC}$ would be the perfect situation to use rituximab.

The safety of rituximab monotherapy has been well established in patients with non-Hodgkin's lymphoma, with the main adverse events being mild to moderate infusion-related reactions occurring after the first infusion. The database of patients having received rituximab in clinical trials and/or clinical practice now includes almost 750000 patients, making rituximab the only antibody in this setting to have such an extensive safety analysis. ${ }^{24}{ }^{25}$ Therefore, we were not surprised at the small number of side effects reported in patients with cryoglobulinemia vasculitis. However, caution is still warranted in this latter indication for the following reasons: no control trial was available, all data came from only small series or case reports, most cases reported on short-term follow-up, and in a large number of cases there was insufficient data to analyse, particularly regarding the course of HCV viral load and liver enzymes. Further assessment of the long-term effects of prolonged B-cell depletion is still required.

We acknowledge some limitations to our analysis. First, there is a potential role that negative publication bias may play in the currently favourable view of rituximab therapy for $\mathrm{MC}$ in the literature. Most studies employing rituximab do not indicate what types of glucocorticoid regimens were used concomitantly with the rituximab. This gives the potentially misleading impression that rituximab alone might have been responsible for the beneficial effects reported. Outside of the two series, the remaining reports are small, and different with regards to patient population, rituximab regimen, outcome definitions, and use of concurrent therapies. Most published cases reported 
Table 2 Main course of cryoglobulinemia vasculitis features after anti-CD20 antibody (rituximab) infusion

\begin{tabular}{|c|c|c|c|}
\hline & $\begin{array}{l}\text { No. of patients } \\
\text { positive at baseline }\end{array}$ & $\begin{array}{l}\text { No. of patients with } \\
\text { available data at follow up }\end{array}$ & $\begin{array}{l}\text { Patients with available } \\
\text { data at follow up (\%) }\end{array}$ \\
\hline \multicolumn{4}{|l|}{ Vasculitis: } \\
\hline Skin involvement & 48 & 40 & \\
\hline $\mathrm{CR}$ & - & 27 & 67.5 \\
\hline PR & - & 5 & 12.5 \\
\hline NR & - & 8 & 20.0 \\
\hline Arthralgia & 35 & 34 & \\
\hline $\mathrm{CR}$ & - & 18 & 52.9 \\
\hline PR & - & 9 & 26.5 \\
\hline NR & - & 7 & 20.6 \\
\hline Neuropathy & 31 & 29 & \\
\hline $\mathrm{CR}$ & - & 9 & 31.0 \\
\hline PR & - & 18 & 62.1 \\
\hline NR & - & 2 & 6.9 \\
\hline Glomerulonephritis & 18 & 18 & \\
\hline CR & - & 12 & 66.6 \\
\hline PR & - & 3 & 16.7 \\
\hline NR & - & 3 & 16.7 \\
\hline Cryoglobulin & 57 & $22^{*}$ & \\
\hline$C R$ & - & 16 & 72.7 \\
\hline PR & - & 2 & 9.1 \\
\hline NR & - & 4 & 18.2 \\
\hline \multicolumn{4}{|l|}{ Follow up after rituximab therapy: } \\
\hline Duration (months), mean (range) & 57 & 56 & $9.7(0.3-24)$ \\
\hline Relapses & - & 14 out of 36 & 39 \\
\hline
\end{tabular}

*The serum cryoglobulin status at the end of follow-up was available in 22 patients.

$\mathrm{CR}$, complete response; NR, non-response; PR, partial response.

on short or mid term follow up, and we need to know on the long-term effects of prolonged B-cell depletion.

\section{CONCLUSION}

Rituximab therapy for patients with mixed cryoglobulinemia vasculitis, HCV-induced or essential, shows a great efficacy on the main vasculitis signs in the majority of reported patients. A relapse of cryoglobulinemia vasculitis was frequently noted. Randomised controlled trials with long-term study are necessary to conclude definitively on the benefit/risk ratio of rituximab therapy in such patients.

Competing interests: None declared.

Table 3 Main course of cryoglobulinemia vasculitis features after anti-CD20 antibody (rituximab) infusion in patients with or without HCV infection

\begin{tabular}{lll}
\hline & HCV + patients $(\mathbf{n}=\mathbf{4 3}), \mathbf{~ n o .}^{*}$ (\%) & HCV - patients $\mathbf{( n = 1 4 ) , ~ n o . * ~ ( \% ) ~}$ \\
\hline Skin involvement & 33 & 7 \\
CR & $24(73)$ & $3(42)$ \\
PR & $3(9)$ & $2(29)$ \\
NR & $6(18)$ & $2(29)$ \\
Arthralgia & 30 & 4 \\
CR & $16(53)$ & $2(50)$ \\
PR & $8(27)$ & $1(25)$ \\
NR & $6(20)$ & $1(25)$ \\
Neuropathy & 25 & 4 \\
CR & $9(36)$ & $0(0)$ \\
PR & $14(56)$ & $4(100)$ \\
NR & $2(8)$ & $0(0)$ \\
Glomerulonephritis & 13 & 5 \\
CR & $9(70)$ & $4(80)$ \\
PR & $2(15)$ & $0(0)$ \\
NR & $2(15)$ & $1(20)$ \\
Cryoglobulin & 15 & 7 \\
CR & $11(73.4)$ & $5(71.4)$ \\
PR & $2(13.3)$ & $0(0)$ \\
NR & $2(13.3)$ & $2(28.6)$ \\
\hline
\end{tabular}

*The reported numbers correspond to patients whose data were available at the end of follow-up.

$\mathrm{CR}$, complete response; NR, non-response; PR, partial response. 


\section{REFERENCES}

1. Gorevic PD, Kassab HJ, Levo Y, Kohn R, Meltzer M, Prose P, et al. Mixed cryoglobulinemia: clinical aspects and long-term follow-up of 40 patients. Am J Med 1980;69:287-308.

2. Brouet JC, Clauvel JP, Danon F, Klein M, Seligmann M. Biologic and clinical significance of cryoglobulins. A report of 86 cases. Am J Med 1974;57:775-88.

3. Cacoub P, Poynard T, Ghillani P, Charlotte F, Olivi M, Piette JC, et al. Extrahepatic manifestations of chronic hepatitis C. MULTIVIRC Group. Multidepartment Virus C. Arthritis Rheum 1999;42:2204-12.

4. Sene D, Limal N, Cacoub P. Hepatitis C virus-associated extrahepatic manifestations: a review. Metab Brain Dis 2004;19:357-81.

5. Misiani R, Bellavita P, Fenili D, Vicari O, Marchesi D, Sironi PL, et al. Interferon alfa2a therapy in cryoglobulinemia associated with hepatitis C virus. N Engl J Med 1994;330:751-6

6. Cacoub P, Saadoun D, Sene D, Limal N, Piette JC. Treatment of hepatitis C virusrelated systemic vasculitis. J Rheumatol 2005;32:2078-82.

7. Cacoub P, Saadoun D, Limal N, Sene D, Lidove O, Piette JC. PEGylated interferon alpha-2b and ribavirin treatment in patients with hepatitis $C$ virus-related systemic vasculitis. Arthritis Rheum 2005;52:911-5.

8. Zaja F, Russo D, Fuga G, Patriarca F, Ermacora A, Baccarani M. Rituximab for the treatment of type II mixed cryoglobulinemia. Haematologica 1999:84:1157-8.

9. Zaja F, De Vita S, Russo D, Michelutti A, Fanin R, Ferraccioli G, et al. Rituximab for the treatment of type II mixed cryoglobulinemia. Arthritis Rheum 2002;46: 2252-4.

10. Arzoo K, Sadeghi S, Liebman HA. Treatment of refractory antibody mediated autoimmune disorders with an anti-CD20 monoclonal antibody (rituximab). Ann Rheum Dis 2002;61:922-4.

11. Zaja F, De Vita S, Mazzaro C, Sacco S, Damiani D, De Marchi G, et al. Efficacy and safety of rituximab in type II mixed cryoglobulinemia. Blood 2003; 101:3827-34

12. Sansonno D, De Re V, Lauletta G, Tucci FA, Boiocchi M, Dammacco F. Monoclonal antibody treatment of mixed cryoglobulinemia resistant to interferon alpha with an anti-CD20. Blood 2003;101:3818-26.

13. Roccatello D, Baldovino S, Rossi D, Mansouri M, Naretto C, Gennaro M, et al. Longterm effects of anti-CD20 monoclonal antibody treatment of cryoglobulinaemic glomerulonephritis. Nephrol Dial Transplant 2004;19:3054-61.
14. Lamprecht $\mathbf{P}$, Lerin-Lozano C, Merz H, Dennin RH, Gause A, Voswinkel J, et al Rituximab induces remission in refractory HCV associated cryoglobulinaemic vasculitis. Ann Rheum Dis 2003;62:1230-3.

15. Ghobrial IM, Uslan DZ, Call TG, Witzig TE, Gertz MA. Initial increase in the cryoglobulin level after rituximab therapy for type II cryoglobulinemia secondary to Waldenstrom macroglobulinemia does not indicate failure of response. Am J Hematol 2004;77:329-30.

16. Ghijsels E, Lerut E, Vanrenterghem Y, Kuypers D. Anti-CD20 monoclonal antibody (rituximab) treatment for hepatitis $\mathrm{C}$-negative therapy-resistant essential mixed cryoglobulinemia with renal and cardiac failure. Am J Kidney Dis 2004;43:e34-8.

17. Catuogno M, Rezai S, Priori R, Magrini L, Valesini G. Serum sickness associated with rituximab in a patient with hepatitis $\mathrm{C}$ virus-related mixed cryoglobulinaemia. Rheumatology (Oxford) 2005;44:406.

18. Basse G, Ribes D, Kamar N, Mehrenberger M, Esposito L, Guitard J, et al. Rituximab therapy for de novo mixed cryoglobulinemia in renal transplant patients. Transplantation 2005;80:1560-4.

19. Koukoulaki M, Abeygunasekara SC, Smith KG, Jayne DR. Remission of refractory hepatitis C-negative cryoglobulinaemic vasculitis after rituximab and infliximab. Nephrol Dial Transplant 2005;20:213-6.

20. Nehme-Schuster H, Korganow AS, Pasquali JL, Martin T. Rituximab inefficiency during type I cryoglobulinaemia. Rheumatology (Oxford) 2005;44:410-1.

21. Quartuccio L, Soardo G, Romano G, Zaja F, Scott CA, De Marchi G, et al. Rituximab treatment for glomerulonephritis in HCV-associated mixed cryoglobulinaemia: efficacy and safety in the absence of steroids. Rheumatology (Oxford) 2006;45:842-6.

22. Cai FZ, Ahern M, Smith M. Treatment of cryoglobulinemia associated periphera neuropathy with rituximab. J Rheumatol 2006:33:1197-8.

23. Saadoun D, Bieche I, Maisonobe T, Asselah T, Laurendeau I, Piette JC, et al. Involvement of chemokines and type 1 cytokines in the pathogenesis of hepatitis $\mathrm{C}$ virus-associated mixed cryoglobulinemia vasculitis neuropathy. Arthritis Rheum 2005; 52:2917-25

24. Ghielmini M, Rufibach K, Salles G, Leoncini-Franscini L, Leger-Falandry C, Cogliatti S, et al. Single agent rituximab in patients with follicular or mantle cell lymphoma: clinical and biological factors that are predictive of response and event-free survival as well as the effect of rituximab on the immune system: a study of the Swiss Group for Clinical Cancer Research (SAKK). Ann Oncol 2005;16:1675-82.

25. Solal-Celigny $\mathbf{P}$. Safety of rituximab maintenance therapy in follicular lymphomas Leuk Res 2006:30(Suppl 1):S16-21.

\section{BNF for Children 2006, second annual edition}

In a single resource:

- guidance on drug management of common childhood conditions

- hands-on information on prescribing, monitoring and administering medicines to children

- comprehensive guidance covering neonates to adolescents

For more information please go to bnfc.org. 